\title{
Ageism from a cross-cultural perspective: results from a national survey of Israelis over the age of $\mathbf{5 0}$
}

\author{
Liat Ayalon ${ }^{1}$ and Ella Cohn-Schwartz ${ }^{2}$ \\ ${ }^{1}$ Louis and Gabi Weisfild School of Social Work, Bar Ilan University, Ramat Gan, Israel \\ ${ }^{2}$ Gerontology Program, Department of Public Health, Ben-Gurion University, Beer Sheva, Israel
}

ABSTRACT

Objectives: We examined differences across three groups in Israel to test the double jeopardy versus the intersection escape hypotheses-which evaluate whether being older and belonging to an underrepresented group serves as a double burden, exposing older minorities to higher levels of perceived ageism or on the contrary, older age serves as a protective factor, especially for underrepresented groups.

Design: A cross-sectional, nationally representative sample, consisting of three groups: Veteran Israelis, Israeli Arabs and immigrants from the Former Soviet Union. The latter two groups represent underrepresented (minority) populations in Israel.

Setting: Community-dwelling Israelis over the age of 50.

Participants: The sample was composed of 1570 participants.

Measurement: To examine the double jeopardy versus the intersectional escape hypothesis, self-perceptions of aging (SPA) and perceived age-based discrimination in the healthcare system were examined as outcome variables.

Results: Among Israeli Arabs, being older was related to better SPA, whereas among the immigrants being older was related to worse SPA. As immigrants became older, they were more likely to report ageist experiences. Israeli Arabs reported higher levels of ageist experiences, regardless of their age.

Conclusions: The findings point to certain groups that require education about ageism and the aging processes and might require further protection from the experiences of ageism. The findings also point to the relevance of different theoretical paradigms that advocate for the consideration of culture and group membership in the understanding of the experiences of ageism.

Keywords: discrimination, self-perceptions of aging, underrepresented group, double jeopardy, culture

\section{Introduction}

Ageism was first coined by Robert Butler over 50 years ago (Butler, 1969). Since then, it has received growing attention given its important public health impact (Levy et al., 2020). Ageism is currently defined as discrimination, prejudice, and stereotypes based on age (Officer and de la FuenteNúñez, 2018). It can be either positive or negative and is directed towards people of various age groups. Ageism can occur at the institutional level as well as at the individual level (Officer and de la FuenteNúñez, 2018). Another important component

Correspondence should be addressed to: Liat Ayalon, Ph.D, Louis and Gabi Weisfild School of Social Work, Bar Ilan University, Ramat Gan 52900, Israel. Phone: 035317910 Email: Liat.ayalon@biu.ac.il. Received 22 Apr 2021; revision requested 15 Jul 2021; revised version received 31 Aug 2021; accepted 07 Sep 2021. First published online 02 November 2021. concerning ageism is the fact that it can be directed towards oneself as well as towards others (Ayalon and Tesch-Römer, 2017).

Ageism is particularly prominent in the healthcare system (Ben-Harush et al., 2017; Wyman et al., 2018), where the present study takes place. Specifically, there is ample research to show that multiple healthcare professionals are inclined to work with younger people rather than older people (Lester et al., 2020). Moreover, multiple studies have shown that when health and social care professionals are presented with similar cases, apart from one's age, they are likely to show a bias towards younger people. Specifically, physicians are less likely to treat older people for suicide risk (Uncapher and Areán, 2000) and more likely to prescribe older people medication, rather than therapy for sexual dysfunction (Gewirtz-Meydan and Ayalon, 2017). 
Moreover, social care professionals are less likely to identify intimate partner violence as abuse in the case of older people and are less likely to offer social interventions (Yechezkel and Ayalon, 2013). Healthcare professionals also are less likely to talk directly to older people, but rather address those who accompany them (Ben-Harush et al., 2017). The impact of ageism does not end here as older people internalize negative stereotypes of old age throughout their lives and these become selfrelevant in old age (Levy, 2009). Past research has shown that negative self-perceptions of aging (SPA) are associated with a variety of negative health and mental health outcomes including increased mortality risk (Ayalon, 2016; Levy et al., 2002a; SargentCox et al., 2014). Moreover, a recent systematic review encompassing 422 studies and over 7 million participants has concluded that the negative effects of ageism on older people's health occur simultaneously at the individual (e.g. self-ageism) and structural levels (e.g. discriminatory practices) in different continents (Chang et al., 2020b).

\section{Ageism intersects with culture}

Like many other social phenomena, the topic of ageism has been examined from cross-national and cross-cultural perspectives (Bergman et al., 2013; Bodner, 2017; North and Fiske, 2015; Vauclair et al., 2017; Wilińska et al., 2018). These perspectives are important as they highlight the role of culture in determining people's attitudes, feelings, and behaviors towards older adults. A recent metaanalysis has found that in contrast to expectations, ageism is more common in Eastern societies than in the Western world (North and Fiske, 2015). This has been attributed to several reasons (North and Fiske, 2015), including modernization processes that have taken place in the East and likely reduce East-West differences, the rapid aging of the population in the East, which might bring with it higher levels of ageism and the limited intergenerational contact found in some Eastern societies (Luo et al., 2013; Woo, 2020). Nonetheless, there are arguments to the contrary that should be considered as well (North and Fiske, 2015). These may include the fact that Eastern societies are more likely to lean towards interdependence and to follow the tradition of filial piety (North and Fiske, 2015; Woo, 2020).

While these studies concern the question of ageism in different societies or cultural contexts, a somewhat different question concerns the intersection of ageism with other cultural/national characteristics, which possibly put some groups at a greater or lesser risk for ageism (Marcus and Fritzsche, 2014). This follows the rationale that age alone is not necessarily a criterion for the experience of ageism, but age in combination with other characteristics might expose people to heightened levels of ageism (Jones et al., 2017). Specifically, the double jeopardy theory argues that possessing more than one devalued status puts people at a greater disadvantage due to the multiplied effect of disadvantages interaction. The theory would suggest that in addition to older age, various social characteristics including cultural origin or underrepresented (minority) affiliation would be the basis for intensified discrimination (Ferraro and Farmer, 1996). According to this theory, people are more likely to be discriminated against if they possess more than a single category that marginalizes them. Hence, being older and belonging to an underrepresented group would result in higher levels of ageism and discrimination (e.g. double jeopardy; Chatters et al., 2020).

Another intersection that has been characterized as double jeopardy is the intersection between older age and immigration status (Raijman and Semyonov, 1997). As older migrants are less likely to be welcomed in the host country due to their age, migration often is restricted to the younger generations, who leave the older generations behind. When migrants grow older, they may experience poorer health, fewer social ties and limited financial resources compared with non-migrants. Thus, their age intersects with their migration status to create a less desired aging trajectory compared with nonmigrants (Dolberg et al., 2018). Consistent with the double jeopardy theory, research has claimed that older women might experience a double disadvantage due to the intersection between old age and women gender, as both characteristics represent disadvantaged statuses (Krekula et al., 2018).

A different claim would argue for an intersectional escape or even a double advantage associated with selected combinations of sociodemographic characteristics (Marcus and Fritzsche, 2014). Specifically, being an older person of an underrepresented group might result in an improved status, even though each of these categories alone (e.g. belonging to an underrepresented group or being an older person) would result in discrimination and a lower status. In support of this hypothesis, research has shown that older women are seen as less threatening and more likable than older men, even though both older people and women belong to lower status categories (Martin et al., 2018).

\section{The present study}

The present study aims to examine the manifestation of ageism in three different Israeli groups: Israeli Jews (e.g. the majority culture), Israeli Arabs, and Israelis who immigrated from the former Soviet 
Union. The latter two groups are considered underrepresented groups in Israeli society. The choice of these three groups stems from the fact that despite the large diversity within each group, they represent a broad division of the main groups in Israel (Brodsky et al., 2018).

Israeli Jews represent $75 \%$ of the population, but over $90 \%$ of the population over the age of 65 . At the ages of 65 and over, only $22 \%$ of Israeli Jews were born in Israel and the remaining were born abroad. Thus, we term this group veteran Israelis to indicate their extended length of time in the country which tends to be associated with multiple advantages, including improved socioeconomic status, health, and physical functioning (Spalter et al., 2014). Israeli Arabs represent the largest underrepresented group and constitute about $20 \%$ of the entire population, but $8 \%$ of those over the age of 65 . It is important to note that the term Israeli Arabs represents a highly diverse group, including Muslims, Christians, Druze, and Bedouins. Each of these groups has its own unique features. The third group represents Israelis from the former Soviet Union. This group constitutes about $23 \%$ of the population over the age of 65 . It is relatively more educated than the general population in Israel and has fewer children relative to veteran Israelis as well as relative to Israeli Arabs (Brodsky, 2018). Despite the high diversity within each of these groups, past research, including large-scale surveys such as the Survey of Health Ageing and Retirement in Europe have employed similar categorization to reflect different groups in Israeli society.

Research on ageism in Israel has found that the rates of ageism are comparable to those reported in other countries. However, in contrast with the general trend which found higher levels of ageism, compared with racism and sexism in multiple European countries, in Israel, the public tends to report higher levels of racism, compared with ageism and sexism (Doron et al., 2011).

Research that has focused on the different groups within Israeli society has found variability in ageism across groups (Bergman et al., 2013). Specifically, a study that examined ageism in Israeli Arabs and Jews among young adults has found that the latter group reports higher levels of ageism compared with Israeli Arabs. Israeli Arabs reported that their culture is more tolerant towards older people and less fearful of them. The study has concluded that older people in the Arab sector enjoy lower levels of ageism due to a more reverent approach towards old age, reflecting more traditional values (Bergman et al., 2013). A different qualitative study, in contrast, was conducted with older Israeli Arabs and their family caregivers. Whereas older Israeli Arabs tended to report high levels of loneliness and a preference towards paid care, arguing that times are changing even in the Arab society, their younger family carers tended to stress the traditional characteristics of the Arab society and their respect and commitment to their elders. The study possibly points to modernization processes in the Arab community in Israel, which are more easily acknowledged by older adults, rather than by younger adults (Ayalon et al., 2015).

To date, research has not examined ageism among Israelis from the former Soviet Union. Nonetheless, research has shown that the living arrangements of this group often consist of older people, living alongside younger people, like the Arab sector in Israel. In addition, both groups are more likely to experience discrimination based on their group membership compared with veteran Israelis (Ayalon, 2019; Epel et al., 2010; Golan-Agnon, 2006; Titzmann et al., 2011).

Considering these contrasting findings, we examined differences across three Israeli groups to test the double jeopardy vs. the intersectional escape/double advantage hypotheses. Hence, we examined whether being older and belonging to an underrepresented group serve as a double burden, exposing older adults who belong to underrepresented groups to higher levels of perceived ageism or on the contrary, serve as protective factors. As research has been inconclusive and quite limited to date, no clear direction to the relationships examined under these research questions is stated.

\section{Methods}

\section{Data and participants}

The sampling frame was developed by a survey agency. Data are based on a national survey of adults aged 50 and above in Israel. Surveys were conducted in Hebrew, Arabic or Russian via the telephone by trained interviewers between April and July, 2020. The sample was composed of 1,570 participants and was representative of the population of adults aged 50 and above in Israel. An ethical approval was obtained by the PI's university and all participants provided a verbal informed consent after learning about the purpose of the study.

\section{Measures}

Self-perceptions of Aging

This measure was used as a proxy of reported of ageism towards oneself. Adults' perceptions of their aging were assessed using the five-items "Attitudes toward aging" subscale of the "Philadelphia Geriatric Center Morale Scale" (Liang and Bollen, 1983). This scale assesses participants' evaluation of their experiences of aging: "Things keep getting worse 
as I get older;" "I have the same energy level as I had last year;" "As I get older, I feel more useless;" "I am happy now as I was when I was younger;" "As I get older, things are better than I thought they'd be." Each item has response options ranging from "Strongly disagree" (1) to "Strongly agree" (6) and a mean score was calculated. The scale had good internal reliability in the current sample $(\alpha=0.75)$.

\section{Perceived age-based discrimination}

We measured experiences of discrimination based on age in the healthcare system (i.e. during visits to doctors, HMOs or hospitals). Respondents were asked how often they had four discriminatory experiences in the healthcare system because of their age in the past year (e.g."being treated with less respect because of your age"). Three of these options were based on the leave-behind questionnaire of the Health and Retirement Survey (Willis et al., 2006). We added an additional variable of "Talking to your companion instead of directly to you," based on previous research on ageism in the Israeli healthcare system (Ben-Harush et al., 2017). We calculated a dummy variable that received 1 if participants reported having any such experience, and 0 if they reported no such experiences. The focus on the healthcare system was geared by the fact that this is one of the most important contexts in which ageism towards older people occurs (Ayalon and Tesch-Römer, 2018).

\section{Group MEMBERSHIP}

Participants were classified into groups based on the language of their interview. Hebrew speakers were tagged as veteran Jews, Arabic speakers were Arab Israelis, and Russian speakers were classified as immigrants from the former Soviet Union. The same classification has been used by the Survey of Health Ageing and Retirement in Europe (http:// igdc.huji.ac.il/englishsite/share/home.aspx) with most studies on group membership in Israel using a similar methodology.

\section{Covariates}

Background information was gathered using age (continuous), gender, and years of education. Participants were also asked to define the state of their health, with responses ranging from 1 ("bad") to 5 ("excellent"). Financial status was assessed by asking about the household's ability to make ends meet financially. We also divided participants into being partnered or not in a partnership. Depression was assessed using the two-item Patient Health Questionnaire (PHQ-2). This measure is comprised of the first two questions of the PHQ- 9, which reflect the core symptoms of depression in the previous 2 weeks (low mood, loss of interest/pleasure; Löwe et al., 2005). Response options for both items range from 1 "Not at all" to 4 "Almost everyday" and the depression score is based on their average. These covariates were selected given their past association with various measures of ageism (Ayalon, 2013; 2018; Diehl et al., 2021).

\section{Data analysis}

We began our analyses with descriptive data of the study sample. We then conducted bivariate analyses of the study variables with the three groups. Chisquare tests measured the bivariate associations with dichotomous variables and ANOVAs with categorical variables. The main stage of analysis was a series of regression models that predicted the two dependent variables (SPA and perceived age-based discrimination) using the independent study variables and the covariates. Each model was run twice - once with the study variables and then with an interaction term of age and group membership. Age was centered at 65 , so that the main effects of the group membership when the interaction is entered will be interpreted as representing the effects among adults aged 65 years old. The interaction between age and group membership was examined to see whether age indeed makes a difference regarding the research questions (e.g. double jeopardy vs. intersectional escape/double advantage). We used OLS regression models to predict SPA. Logistic regression models predicted perceived age-based discrimination, as it was a binary variable.

\section{Results}

Table 1 shows the sample characteristics. Twentyeight percent of the sample reported any experience of discrimination in healthcare. Their selfperceptions of aging were somewhat positive (4 of 6). In terms of groups, most of the sample was composed of veteran Jews, almost one fifth were Israeli Arabs, and a minority were immigrants. The sample mean age was 64 and almost half were women. They were relatively well-educated with an average of 13 years of education. They rated their financial status and health as medium and over three quarters were partnered. They had a relatively low depression score.

Supplemental Table 1 shows the bivariate associations of the study variables with the group membership. Group differences were seen in relation to SPA - participants from both underrepresented groups reported worse SPA compared to participants from the majority group. In addition, participants from the underrepresented groups reported experiencing significantly more age-based 
Table 1. Sample characteristics of the study

\begin{tabular}{|c|c|c|c|}
\hline VARIABLE & MEAN (SD) & $\%$ & RANGE \\
\hline Perceived age-based discrimination & & $444(28.28 \%)$ & \\
\hline SPA & $4.13(1.26)$ & & $1-6$ \\
\hline Age & $64.16(9.39)$ & & $50-96$ \\
\hline Population: Veteran Jews & & $1183(75.35 \%)$ & \\
\hline Population: Arab Israelis & & $283(18.03 \%)$ & \\
\hline Population: USSR immigrants & & $104(6.62 \%)$ & \\
\hline Gender: Women & & $752(47.90 \%)$ & $1-2$ \\
\hline Years of education & $13.47(4.65)$ & & $0-30$ \\
\hline Financial status & $2.44(0.99)$ & & $1-4$ \\
\hline Partnered & & $1164(74.9 \%)$ & \\
\hline Self-rated health & $3.06(1.12)$ & & $1-5$ \\
\hline Depression & $1.60(0.83)$ & & $1-4$ \\
\hline
\end{tabular}

Note. SPA $=$ Self-perceptions of aging.

Table 2. Linear regression models predicting self-perceptions of aging

\begin{tabular}{|c|c|c|c|c|c|c|}
\hline \multirow[b]{2}{*}{ Variable } & \multicolumn{6}{|c|}{ SPA } \\
\hline & B & SE & $B E T A$ & B & SE & $B E T A$ \\
\hline Group: Arab Israelis ${ }^{1}$ & -0.54 & 0.10 & $-0.16^{\text {**** }}$ & -0.55 & 0.10 & $-0.17^{* * *}$ \\
\hline Group: immigrants from the former Soviet Union ${ }^{1}$ & -0.47 & 0.12 & $-0.09^{* * *}$ & -0.34 & 0.13 & $-0.07^{* *}$ \\
\hline Age & 0.00 & 0.00 & -0.02 & -0.01 & 0.00 & -0.04 \\
\hline Gender: Women & 0.17 & 0.06 & $0.07^{* *}$ & 0.15 & 0.06 & $0.06^{*}$ \\
\hline Education (years) & -0.01 & 0.01 & -0.03 & -0.01 & 0.01 & -0.03 \\
\hline Financial status & 0.10 & 0.03 & $0.08^{* *}$ & 0.10 & 0.03 & $0.08^{* *}$ \\
\hline Partnered & 0.17 & 0.07 & $0.06^{*}$ & 0.17 & 0.07 & $0.06^{*}$ \\
\hline Self-rated health & 0.23 & 0.03 & $0.20 * * *$ & 0.24 & 0.03 & $0.21^{* * *}$ \\
\hline Depression & -0.56 & 0.04 & $-0.37^{* * *}$ & -0.55 & 0.04 & $-0.36^{* * *}$ \\
\hline \multicolumn{7}{|l|}{ Interactions: } \\
\hline Age $\times$ Arab Israelis & & & & 0.02 & 0.01 & $0.07^{* *}$ \\
\hline Age $\times$ immigrants from the former Soviet Union & & & & -0.03 & 0.01 & $-0.05^{*}$ \\
\hline$R^{2}$ & & & 0.28 & & & 0.29 \\
\hline
\end{tabular}

Note. $^{*} p<.05,{ }^{* *} p<.01,{ }^{* * *} p<.001 ;$ SPA $=$ Self-perceptions of aging.

${ }^{1}$ Reference: Veteran Jews.

discrimination in healthcare settings. Specifically, $54 \%$ of Arab Israelis and $47 \%$ of the immigrants reported such experiences, while only $20 \%$ of veteran Jews had such reports. Additional patterns of differences emerged between the groups. Immigrants were older and more educated, whereas veteran Jews reported the best financial status and rated their health as best. Interestingly, Arab Israelis reported the least depression. The groups did not differ in relation to gender and partnership status.

The main stage of the analysis was the regression models. Table 2 presents the regression model predicting SPA. Adults from both underrepresented groups reported worse SPA compared to the majority group. The interaction with age was found to be significant in a significance test comparing the nested models with main effects and with the addition of the interaction $(F(2,1,435)=5.84$, $p=0.003)$. The interaction terms were significant for both underrepresented groups, but in opposite directions. Among the Israeli Arabs, being older was related to better SPA, whereas among the immigrants being older was related to worse SPA. The interaction is presented graphically in Figure 1.

Table 3 presents a logistic regression predicting perceived age-based discrimination. It shows that both underrepresented groups had a higher likelihood of experiencing age-based discrimination compared to the majority group. The interaction with age was found to be significant in a significance test comparing the nested models with main effects and with the addition of the interaction $\left(\chi^{2}(2,1435)=9.12, p=0.01\right)$. The immigrants had an interaction with age, such that as they became 
Table 3. Logistic regression models predicting perceived age-based discrimination

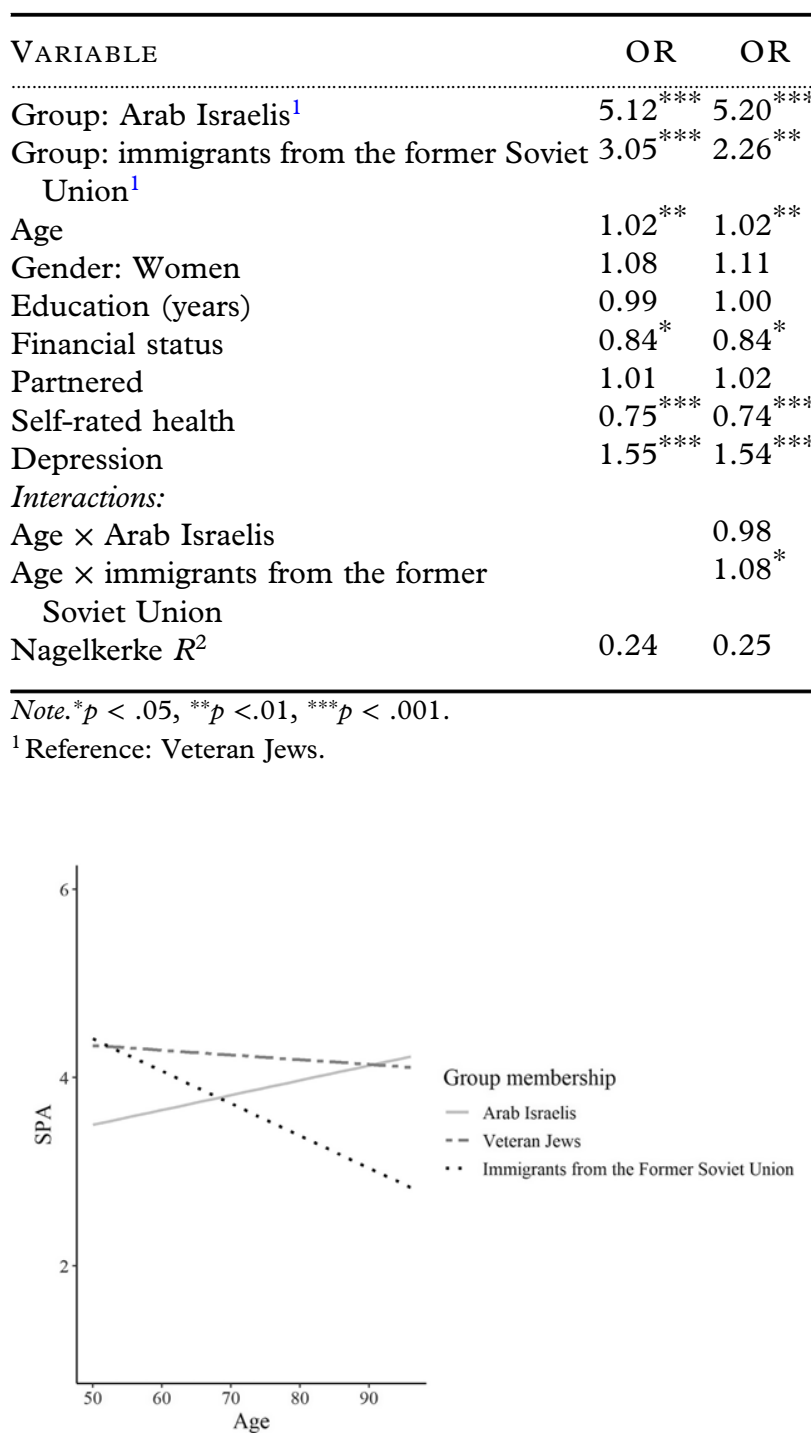

Figure 1. Self-perceptions of aging as a function of the interaction between population group membership and age.

older, they were more likely to report ageist experiences. Figure 2 presents the logistic regression curve which indicates the probability of experiencing age-based discrimination among the three groups of various ages. We note that the interaction terms added only one additional percent to the variance explained by the regression models. They serve, therefore, mainly to underscore the dynamics of the inter-relationship between age and group membership in relation to ageism.

\section{Discussion}

Ageism does not take place in a vacuum. Instead, it occurs within a social context in which cultural

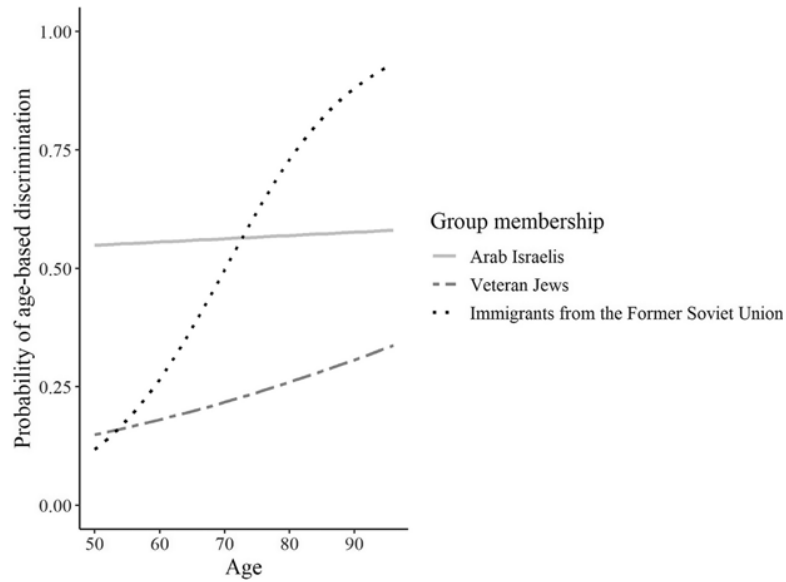

Figure 2. Perceived age-based discrimination as a function of the interaction between population group membership and age.

beliefs and values likely play a role (Bodner, 2017; Wilińska et al., 2018). Moreover, it is not age alone, which affects people's experiences of discrimination, but instead age intersects with other characteristics (Marcus and Fritzsche, 2014). The present study is set to examine research questions, which are not only theoretical in nature, but also of important practical implications. As ageism plays a major role in people's life experiences and has major public health implications (Chang et al., 2020a; Levy et al., 2020), it is important to better understand the social contexts and personal characteristics that are of relevance to people's experiences of ageism. Relying on the large diversity of Israeli society, we examined ageist beliefs as well as perceived ageist practices in three different groups: the majority group of veteran Israelis, Israeli Arabs, and Israelis from the former Soviet Union.

Our findings suggest that indeed, group membership plays a role in people's reports of self-ageism. Specifically, for SPA, there was a significant interaction between group membership and age. For Arab Israelis, as age increased, SPA improved. For Israelis from the former Soviet Union, on the other hand, as age increased, SPA became increasingly worse. The question concerning the role of group membership in people's SPA is not only theoretical. Past research has shown that SPA plays a major role in people's health, well being, ability to cope with various health problems and even mortality (Ayalon, 2016; Levy and Myers, 2004; Levy et al., 2002b). Hence, the group differences identified in this study possibly allude to those groups that require further education and support concerning their aging process.

The Arab population has been portrayed in past research as a more traditional, filial society, which respects its elders (Bergman et al., 2013). As such, it is possible that as people age, their self-perceptions 
of their own aging improve. Hence, they possibly experience advantageous aging as they reach old age. In contrast, Israelis from the former Soviet Union experienced migration in older age and often were forced to pursue employment paths for which they were over qualified (Getta, 2020). In old age, it is possible that past experiences of migration play a role and result in lower SPA. This follows the cumulative disadvantages theory, which suggests that multiple negative experiences accumulate in old age (Ferraro et al., 2009). Hence both the double jeopardy and the intersectional escape/double advantage hypotheses are supported here. When reviewing these findings, however, it is important to acknowledge the fact that SPA may reflect different experiences for middle-aged respondents, who view SPA as a future-oriented expectation, whereas older people, report their SPA based on their current aging experiences. Hence, even though the same measure is used to assess SPA across different age groups, the construct is interpreted differently in different age groups.

As for perceived age-based discrimination, our findings support the double jeopardy hypothesis by showing that both Arab Israelis and Israelis from the former Soviet Union report higher levels of discrimination in the health care system. Arab Israelis were five times more likely to report discrimination, compared to the veteran Jews, and immigrants from the former Soviet Union were three times more likely to experience discrimination. Moreover, Israelis from the former Soviet Union reported higher levels of perceived ageism in the healthcare system as their age increase, suggesting that the experience of perceived age discrimination intensifies with age. This was not the experience of Israeli Arabs who reported higher levels of discrimination regardless of their age. We note that the use of the age-based interaction term added only one additional percent to the variance explained by the regression. It serves, therefore, mainly to underscore the dynamics of the interrelationship between age and group membership in relation to age-based discrimination.

Israelis from the former Soviet Union represent a unique group of migrants who are better qualified and educated than the majority culture yet migrated at a later stage in life and as a result were not adequately absorbed in the country. The fact that these individuals chose to be interviewed in Russian more than 20 years after the large immigration wave had ended further alludes to their limited integration in the country. This group is likely disadvantaged due to its migration status as well as old age (Dolberg et al., 2018). Hence, as the age of members in this group increases, they likely report higher levels of perceived age-based discrimination. Similar to Israelis from the former Soviet Union (Titzmann et al., 2011), Israeli Arabs also are exposed to high levels of discrimination in Israeli society (GolanAgnon, 2006). Even more specifically, past research has shown that Israeli Arabs perceive high levels of discrimination in the context of elder care (Ayalon, 2019). Whereas that study has found that they tended to assign the reasons for discrimination to their minority status, the present study adds by showing that sense of discrimination also is attributed to age, thus possibly stressing the relevance of the double jeopardy hypothesis.

In reviewing the study findings, it is important to consider its limitations. First, we did not evaluate other types of discrimination. Thus, we can talk about the double jeopardy hypothesis in relation to ageism, but not in relation to other prominent "isms" including sexism or racism. Second, as already noted, our selection of groups was based on their preferred language. The choice not to speak Hebrew, but rather Russian or Arabic points to a tendency to disassociate from the majority culture, which speaks Hebrew. Our findings might have been different had we included people of these population groups, regardless of their preferred language. This, however, is the common practice in other nationally representative studies conducted in Israel, such as the Survey of Health Ageing and Retirement in Europe (Litwin and Sapir, 2008). Another fact that should be noted is the small effect size of the interaction effects identified in this study. This suggests that the interpretation of these interactions should be done with cautious, with the understanding that statistical significance is not equal to clinical significance.

Despite its limitations, the study has several strengths that should be noted. Relying on a large, representative sample of people over the age of 50, this study examined the phenomenon of ageism from different theoretical perspectives. Our findings show that older Israeli Arabs tend to report better SPA as they grow older, whereas older Israelis from the former Soviet Union are more likely to report worse SPA, as they grow older. Moreover, older migrants from the former Soviet Union are particularly likely to report high levels of perceived agebased discrimination, as they grow older, whereas Israeli Arabs are likely to report higher perceived age-based discrimination regardless of their age. The findings point to certain groups that require education about ageism and the aging processes and might require further protection from the experiences of self-ageism and ageism. The findings also point to the relevance of different theoretical paradigms that advocate for the consideration of culture and group membership in the understanding of the experiences of ageism. As the groups examined in this study are largely heterogenous in nature, future 
research will benefit from the more refined categorization of group membership to better understand this heterogeneity. In addition, qualitative research can provide important insights into the findings by exploring their meaning in more nuanced ways.

\section{Conflict of interest}

None.

\section{Description of author(s)' roles}

LA: concept development, drafting of the manuscript, and critical revisions; ECS: concept development, data analysis, and critical revisions.

\section{Acknowledgement}

The study was funded by the Israel National Institute for Health Policy Research 2018/4r.

\section{Supplementary material}

To view supplementary material for this article, please visit https://doi.org/10.1017/S1041610221001241

\section{References}

Ayalon, L. (2013). Feelings towards older vs. younger adults: results from the European Social Survey. Educational Gerontology, 39, 888-901.

Ayalon, L. (2016). Satisfaction with aging results in reduced risk for falling. International Psychogeriatrics, 28,741 .

Ayalon, L. (2018). Perceived age discrimination: a precipitator or a consequence of depressive symptoms? The Fournals of Gerontology: Series B, 73, 860-869.

Ayalon, L. (2019). Perceived discrimination and stigma in the context of the long-term care insurance law from the perspectives of Arabs and the Jews in the North of Israel. International Fournal of Environmental Research and Public Health, 16, 3511.

Ayalon, L., Karkabi, K., Bleichman, I., Fleischmann, S. and Goldfracht, M. (2015). Between modern and traditional values: informal mental health help-seeking attitudes according to Israeli Arab women, primary care patients and their providers. International fournal of Social Psychiatry, 61, 386-393.

Ayalon, L. and Tesch-Römer, C. (2017). Taking a closer look at ageism: self-and other-directed ageist attitudes and discrimination. European fournal of Ageing, 14, 1-4.

Ayalon, L. and Tesch-Römer, C. (2018). Contemporary perspectives on ageism: Berlin: Springer.

Ben-Harush, A. et al. (2017). Ageism among physicians, nurses, and social workers: findings from a qualitative study. European fournal of Ageing, 14, 39-48.
Bergman, Y. S., Bodner, E. and Cohen-Fridel, S. (2013). Cross-cultural ageism: ageism and attitudes toward aging among Jews and Arabs in Israel. International Psychogeriatrics, 25, 6-15.

Bodner, E. (2017). 10 Cross-Cultural Differences in Ageism. In: T.D. Nelson (Ed), Ageism: stereotyping and prejudice against older persons, 291. Boston, Massachusets: MIT University Press

Brodsky, J., Snor, Y., Beer, S., Newil, A. (2018). $65+$ in Israel. JDC Brookdale. Available online: https://brookdale .jdc.org.il/publication/statistical-abstract-elderly-israel2018/

Butler, R. N. (1969). Age-Ism: Another form of bigotry. The Gerontologist, 9, 243-246.

Chang, E. S., Kannoth, S., Levy, S., Wang, S.-Y., Lee, J. E. and Levy, B. R. (2020b). Global reach of ageism on older persons' health: a systematic review. PLOS ONE, 15, e0220857.

Chang, E.-S., Kannoth, S., Levy, S., Wang, S.-Y., Lee, J. E. and Levy, B. R. (2020a). Global reach of ageism on older persons' health: A systematic review. PLOS ONE, 15, e0220857.

Chatters, L. M., Taylor, H. O. and Taylor, R. J. (2020). Older Black Americans during COVID-19: Race and age double jeopardy. Health Education $\mathcal{G}$ Behavior, 47, 855-860.

Diehl, M., Wettstein, M., Spuling, S. M. and Wurm, S. (2021). Age-related change in self-perceptions of aging: Longitudinal trajectories and predictors of change. Psychology and Aging, 36(3), 344-359.

Dolberg, P., Sigurðardóttir, S. H. and Trummer, U. (2018). Ageism and older immigrants. In: L. Ayalon and C. Tesch-Römer (Eds.), Contemporary perspectives on ageism (pp. 177-191). Berlin: Springer.

Doron, I., Ayalon, L., Shiovitz-Ezra, S., Bodner, E., Inbar, N., Koren, C., Golander, H., Hazan, H., Alon, S., Brodsky, J., and Goldberg. A. (2011). Ageism in Israel and Europe: Preliminary findings from the European Social Survey (ESS). Gerontology, 38, 73-92 (in Hebrew).

Epel, O. B., Kaplan, G. and Moran, M. (2010). Perceived discrimination and health-related quality of life among Arabs and Jews in Israel: a population-based survey. $B M C$ Public Health, 10, 1-9.

Ferraro, K. F. and Farmer, M. M. (1996). Double jeopardy to health hypothesis for African Americans: analysis and critique. Fournal of Health and Social Behavior, 27-43.

Ferraro, K. F., Shippee, T. P. and Schafer, M. H. (2009). Cumulative inequality theory for research on aging and the life course. In: V. L. Bengston, D. Gans, N. M. Pulney, and M. Silverstein (Eds.), Handbook of theories of aging (pp. 413-433). Berlin: Springer Publishing Company.

Getta, M. (2020). The integration challenge of Russianspeaking Jews in Israel. Available online: https://dspace.cuni .cz/handle/20.500.11956/121365

Gewirtz-Meydan, A. and Ayalon, L. (2017). Physicians' response to sexual dysfunction presented by a younger vs. an older adult. International Fournal of Geriatric Psychiatry, 32, 1476-1483.

Golan-Agnon, D. (2006). Separate but not equal: discrimination against Palestinian Arab students in Israel. American Behavioral Scientist, 49, 1075-1084. 
Jones, K. P., Sabat, I. E., King, E. B., Ahmad, A., McCausland, T. C. and Chen, T. (2017). Isms and schisms: a meta-analysis of the prejudice-discrimination relationship across racism, sexism, and ageism. Fournal of Organizational Behavior, 38, 1076-1110.

Krekula, C., Nikander, P. and Wilińska, M. (2018). Multiple marginalizations based on age: gendered ageism and beyond. In: L. Ayalon and C. Tesch-Römer (Eds.), Contemporary perspectives on ageism (pp. 33-50). Berlin: Springer.

Lester, P. E., Dharmarajan, T. and Weinstein, E. (2020). The looming geriatrician shortage: Ramifications and solutions. Fournal of Aging and Health, 32, 1052-1062.

Levy, B. (2009). Stereotype embodiment: A psychosocial approach to aging. Current Directions in Psychological Science, $18,332-336$.

Levy, B. R. and Myers, L. M. (2004). Preventive health behaviors influenced by self-perceptions of aging. Preventive Medicine, 39, 625-629.

Levy, B. R., Slade, M. D., Chang, E.-S., Kannoth, S. and Wang, S.-Y. (2020). Ageism amplifies cost and prevalence of health conditions. The Gerontologist, 60, 174-181.

Levy, B. R., Slade, M. D. and Kasl, S. V. (2002a). Longitudinal benefit of positive self-perceptions of aging on functional health. The fournals of Gerontology Series B: Psychological Sciences and Social Sciences, 57, P409-P417.

Levy, B. R., Slade, M. D., Kunkel, S. R. and Kas1, S. V. (2002b). Longevity increased by positive self-perceptions of aging. fournal of Personality and Social Psychology, 83, 261.

Liang, J. and Bollen, K. A. (1983). The structure of the Philadelphia Geriatric Center Morale scale: a reinterpretation. Fournal of Gerontology, 38, 181-189.

Litwin, H. and Sapir, E. V. (2008). 3.4 Israel: Diversity Among Population Groups. In: First Results from the Survey of Health, Ageing and Retirement in Europe (2004-2007): Starting the Longitudinal Dimension (p. 93). Mannheim: Mannheim Research Institute for the Economics of Aging.

Löwe, B., Kroenke, K. and Gräfe, K. (2005). Detecting and monitoring depression with a two-item questionnaire (PHQ-2). Fournal of Psychosomatic Research, 58, 163-171.

Luo, B., Zhou, K., Jin, E. J., Newman, A. and Liang, J. (2013). Ageism among college students: A comparative study between US and China. Fournal of Cross-Cultural Gerontology, 28, 49-63.

Marcus, J. and Fritzsche, B. A. (2014). One size doesn't fit all: toward a theory on the intersectional salience of ageism at work. Organizational Psychology Review, 5, 168-188.
Martin, A. E., North, M. S. and Phillips, K. W. (2018). Intersectional escape: older women elude agentic prescriptions more than older men. Personality and Social Psychology Bulletin, 45, 342-359.

North, M. S. and Fiske, S. T. (2015). Modern attitudes toward older adults in the aging world: a cross-cultural meta-analysis. Psychological Bulletin, 141, 993-1021.

Officer, A. and de la Fuente-Núñez, V. (2018). A global campaign to combat ageism. Bulletin of the World Health Organization, 96, 295-296.

Raijman, R. and Semyonov, M. (1997). Gender, ethnicity, and immigration: Double disadvantage and triple disadvantage among recent immigrant women in the Israeli labor market. Gender E Society, 11, 108-125.

Sargent-Cox, K. A., Anstey, K. J. and Luszcz, M. A. (2014). Longitudinal change of self-perceptions of aging and mortality. Fournals of Gerontology Series B: Psychological Sciences and Social Sciences, 69, 168-173.

Spalter, T., Brodsky, J. and Shnoor, Y. (2014). Improvements and decline in the physical functioning of Israeli older adults. The Gerontologist, 54, 919-929.

Titzmann, P. F., Silbereisen, R. K., Mesch, G. S. and Schmitt-Rodermund, E. (2011). Migration-specific hassles among adolescent immigrants from the former Soviet Union in Germany and Israel. Fournal of CrossCultural Psychology, 42, 777-794.

Uncapher, H. and Areán, P. A. (2000). Physicians are less willing to treat suicidal ideation in older patients. Fournal of American Geriatric Society, 48, 188-192.

Vauclair, C.-M., Hanke, K., Huang, L.-L. and Abrams, D. (2017). Are Asian cultures really less ageist than Western ones? It depends on the questions asked. International fournal of Psychology, 52, 136-144.

Wilińska, M., de Hontheim, A. and Anbäcken, E.-M. (2018). Ageism in a Cross-Cultural Perspective: Reflections from the Research Field. In L. Ayalon and C. TeschRömer (Eds.) Contemporary Perspectives on Ageism (pp. 425-440). Berlin: Springer.

Woo, J. (2020). The myth of filial piety as a pillar for care of older adults among Chinese populations. Advances in Geriatric Medicine and Research, 2(2).

Wyman, M. F., Shiovitz-Ezra, S. and Bengel, J. (2018). Ageism in the health care system: Providers, patients, and systems. In: L. Ayalon and C. Tesch-Römer (Eds.), Contemporary perspectives on ageism (pp. 193-212). Berlin: Springer.

Yechezkel, R. and Ayalon, L. (2013). Social workers' attitudes towards intimate partner abuse in younger vs. older women. Fournal of Family Violence, 28, 381-391. 